\title{
Active Flatfoot Phenomenon Caused by Posterior Tibial Tendon Dys- function
}

\author{
Tomomasa Nakamura $^{1}$, Ichiro Sekiya ${ }^{1}$, Takeshi Muneta ${ }^{1}$ and Haruyasu Yamamoto ${ }^{1,2}$ \\ ${ }^{1}$ Department of Orthopedic Surgery, Tokyo Medical and Dental University, Tokyo, Japan \\ ${ }^{2}$ Chiba Kashiwa Rehabilitation Hospital, Chiba, Japan
}

*Corresponding author: Tomomasa Nakamura, Department of Orthopedic Surgery, Tokyo Medical and Dental University, Tokyo, Japan, E-mail: nakamorj@gmail.com

\begin{abstract}
Background: Posterior Tibial Tendon Dysfunction (PTTD) is considered the major cause of adult acquired flatfoot deformity. We report a case of PTTD showing "active flatfoot" phenomenon in which a patient can voluntarily transition from a normal foot shape to flatfoot deformity.

Case: The patient was a 52-year-old female who could voluntarily provoke flatfoot deformity by dorsiflexion of her left ankle. Her chief complaint was medial ankle pain, which we diagnosed as stage II of the posterior tibial tendon dysfunction according to the physical and radiographic examination. The Japanese Society for Surgery of the Foot (JSSF) midfoot score was 66 points. Her hind foot showed slight valgus deformity in a usual weight bearing position, but she could voluntarily provoke flatfoot deformity during active dorsiflexion with crepitus in both standing and supine positions. We termed this physical finding "active flatfoot".

Results: Conservative treatments, which included a shoe insert, were not effective. We performed the medial displacement calcaneal osteotomy, the transfer of the flexor digitalis longus to the navicular and the shortening of the tibial posterior tendon. Surgical findings demonstrated that the posterior tibial tendon was swollen, elongated and degenerated. Two years after operation, her medial ankle pain was resolved, the alignment of tarsal bones on the radiographs was improved, and the JSSF midfoot score was increased to 100 points. Furthermore, it became impossible for the patient to provoke "active flatfoot".

Conclusion: We revealed the unusual phenomenon that could not be covered by Myerson classification by careful interview and dynamic observation. It is necessary to make careful dynamic examination for understanding accurate pathology.
\end{abstract}

\section{Keywords}

Posterior tibial tendon dysfunction, Acquired flatfoot deformity, Surgical treatment, Voluntary deformity

\section{Introduction}

Adult acquired flatfoot deformity is characterized by the loss of medial arch height, valgus deformity of calcaneus and forefoot abduction while standing [1-3]. Posterior Tibial Tendon Dysfunction (PTTD) is considered the major cause of this clinical state. PTTD was classified from the physiological and $\mathrm{X}$-ray findings by Johnson and Strom [4] and modified by Myerson [5]. The deformity is evaluated in standing position and dynamically examined in lying or sitting position for determining whether the deformity is flexible or rigid. Here, we report a case of "active flatfoot" in which a patient can voluntarily transition from a normal foot alignment to flatfoot.

\section{Case Report}

The patient was a 52-year-old female who had been complaining of left medial ankle pain for one year. She was treated conservatively at another hospital, but gradually became troubled by the valgus deformity of the heel that suddenly occurred while walking. In clinical findings during weight bearing positions, the posterior tibial tendon was swollen, the longitudinal arch was slightly decreased, and the hind foot showed slight valgus deformity. In addition, too many toes sign and single heel rise test were positive. Interestingly, while her ankle voluntarily positioned into dorsiflexion, the hind foot moved valgus and the forefoot abducted, and provoked flatfoot deformity with crepitus (Figure 1 and Supplementary Movie 1). In non-weight bearing condition, she was able to prevent or provoke this phenomenon intentionally. 
A

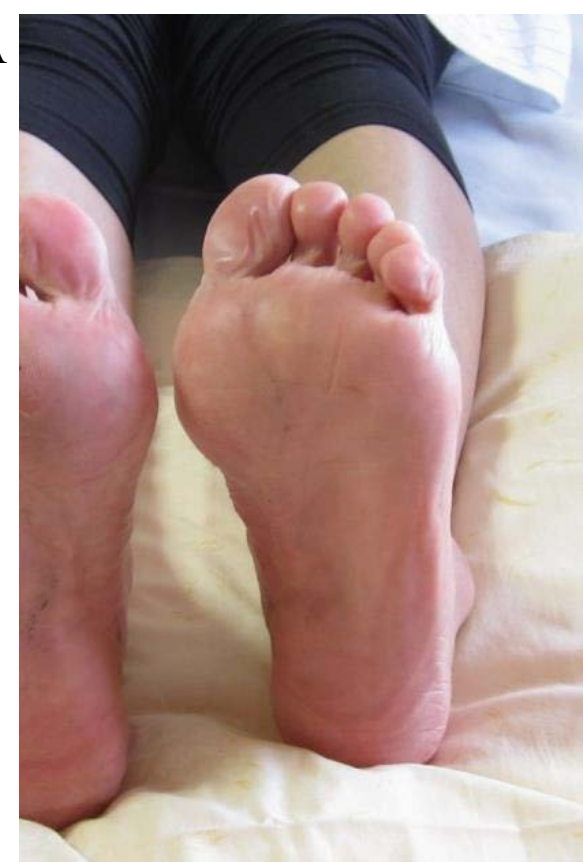

B

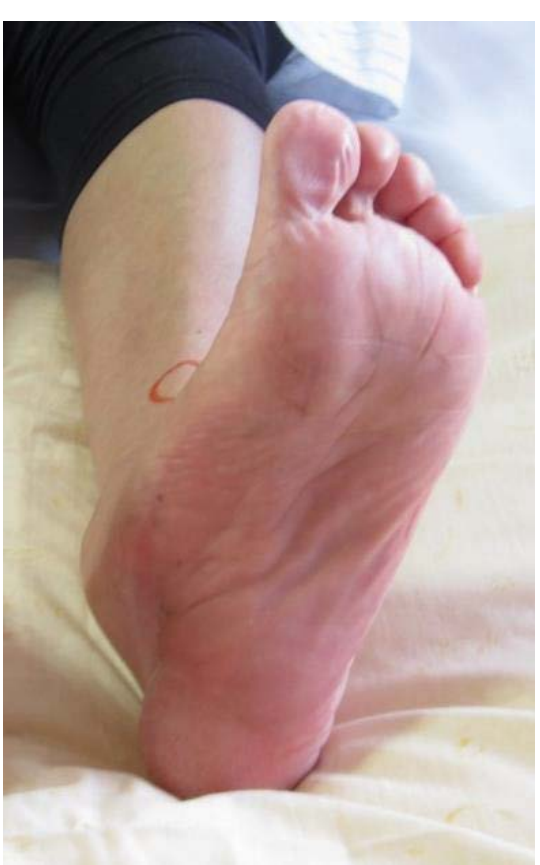

Figure 1: Active flat foot phenomenon A) The appearance of the plantar at rest; B) In active dorsiflexion, the forefoot pronated and adducted.
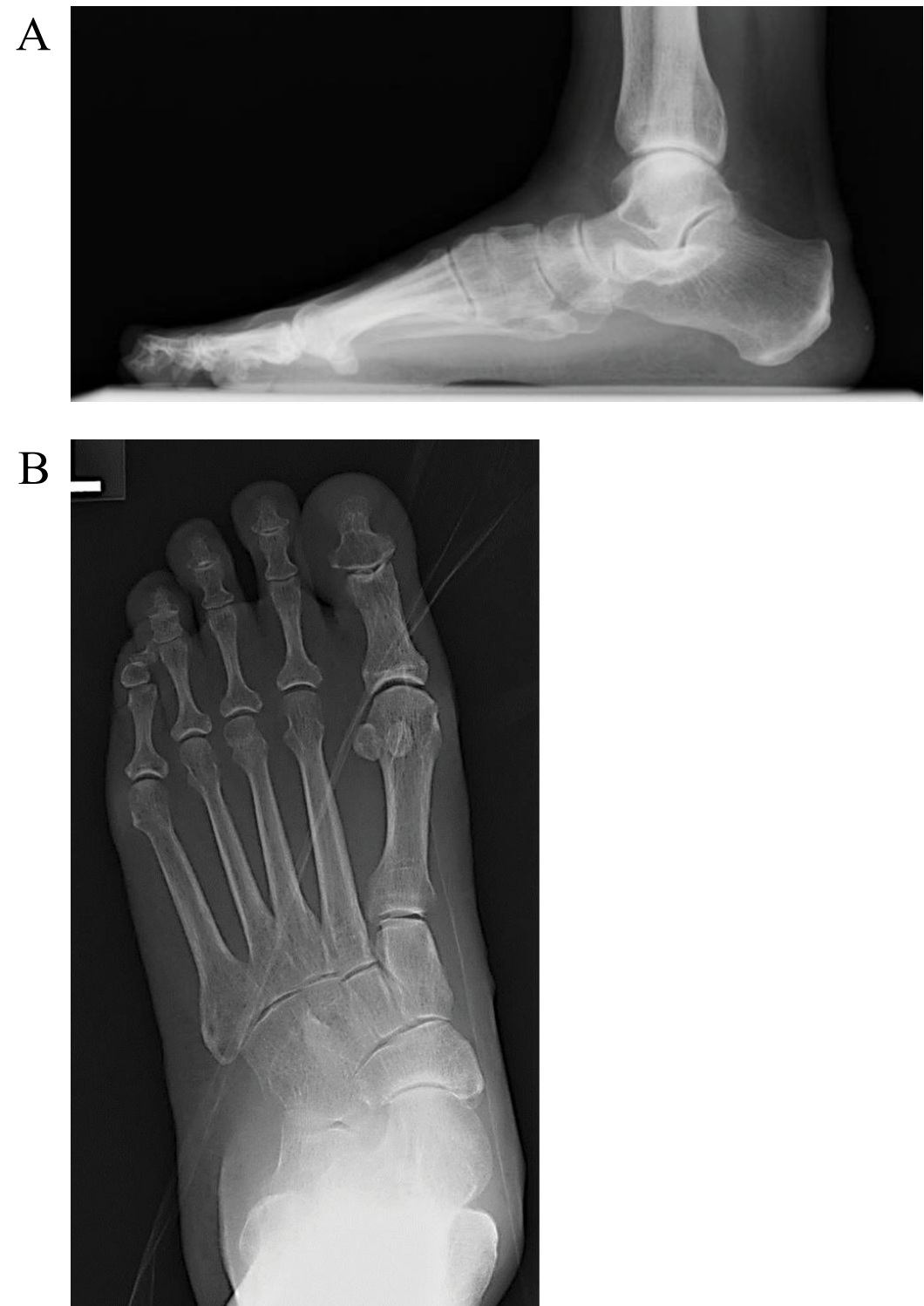

Figure 2: Radiographs before surgery in weight bearing position A) Lateral view; B) Dorsoplantar view. 
We termed this physical finding "active flatfoot". On the lateral radiograph, calcaneal pitch was $20^{\circ}$ on the left foot and $22^{\circ}$ on the right foot, and talo-first metatarsal angle was $20^{\circ}$ on the left foot and $5^{\circ}$ on the right foot. On the dorsoplantar view the talonavicular coverage angle was $40^{\circ}$ on the left foot and $28^{\circ}$ on the right foot (Figure 2). The Japanese Society for Surgery of the Foot (JSSF) midfoot score $[6,7]$ was 66 points. According to these findings, we diagnosed her left foot as PTTD stage II. The patient used the University of California Biomechanics Laboratory (UCBL) shoe insert for three months, but ceased to do so when it became too painful.

Then, we performed the medial displacement calcaneal osteotomy, the transfer of the flexor digitalis long us to the navicular and the shortening of the posterior tibial tendon. Surgical findings demonstrated that the posterior tibial tendon was swollen, elongated and degenerated (Figure 3A). Histopathological examination showed degeneration in this posterior tibial tendon (Figure 3B).

The below-knee casting was performed in the ankle $30^{\circ}$ planter flexion and a slightly varus position postoperatively. The casting was removed two months after the operation, and then full weight bearing was allowed. One year after the initial surgery, two screws used for fixation of osteotomy were removed.
Two years after the initial surgery, her medial ankle pain had disappeared, and the JSSF midfoot score increased to 100 points. The latest lateral radiograph of the left foot showed that calcaneal pitch was $19^{\circ}$ and talo-first metatarsal angle was $-3^{\circ}$ on the left foot. Additionally, on the dorsoplantar radiograph of the left foot the talonavicular coverage angle was $22^{\circ}$ (Figure 4). Her left foot showed no flatfoot deformity while standing and she has become able to tiptoe. Furthermore, she could no longer provoke "active flatfoot".

\section{Discussion}

There is no report of active flatfoot in which a patient voluntarily transitions from a normal foot position to flatfoot. At first, we did not discover this phenomenon through a usual examination. In the corresponding interview the patient complained of an unpleasant, self-shifting phenomenon during active range of motion of the ankle in addition to pain on the medial side of the foot. Furthermore, she demonstrated the ability to achieve flatfoot deformity with crepitus by herself.

In this case report, our careful interview and observation revealed a novel pathological condition that cannot be covered by Myerson classification, the most prevailing one for adult acquired flatfoot. Our case seemed close to Myerson's classification stage II, defined as a
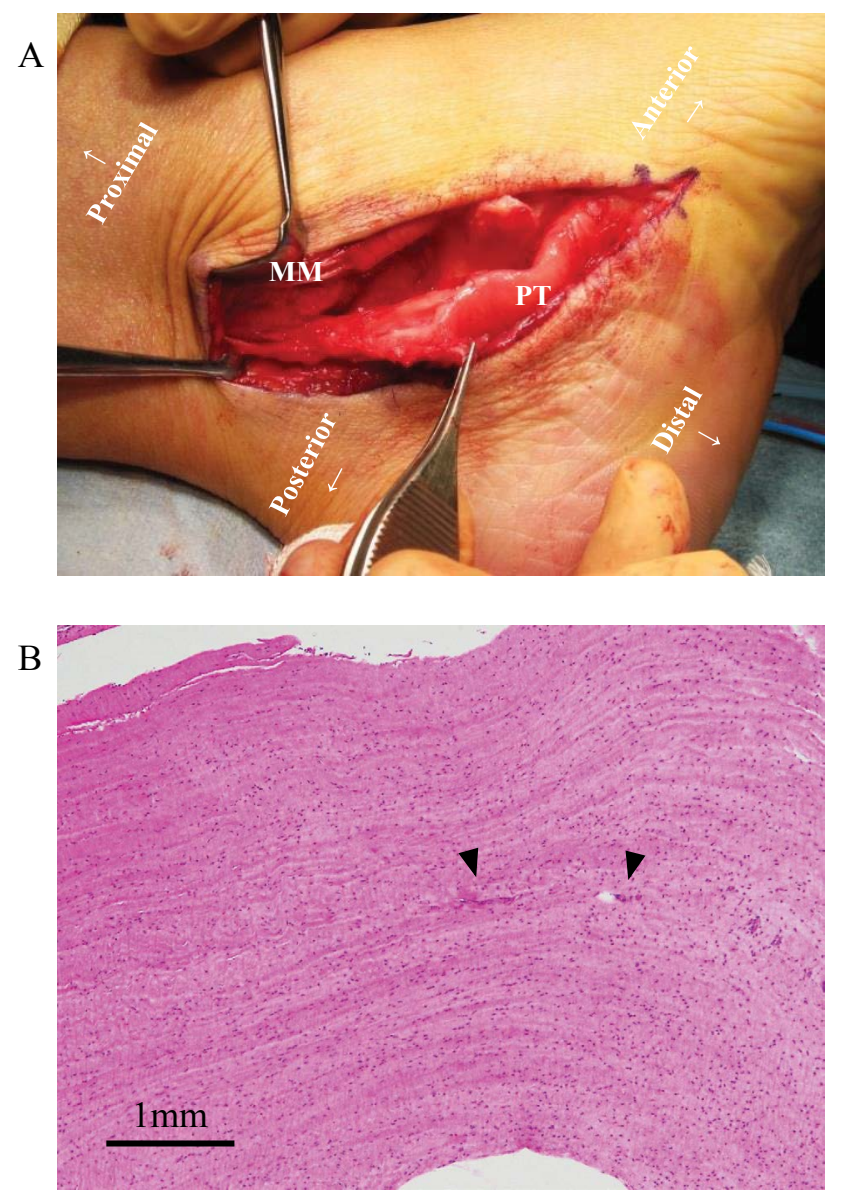

Figure 3: Surgical and histological findings of posterior tibial tendon A) Intraoperative photograph demonstrating the swollen and elongated posterior tibial tendon. PT: Posterior Tibial Tendon; MM: Medial Malleolus of the ankle; B) Histological feature showing irregular collagen fibril and cell, increased cell density, and neovascularization (arrowhead). 
A

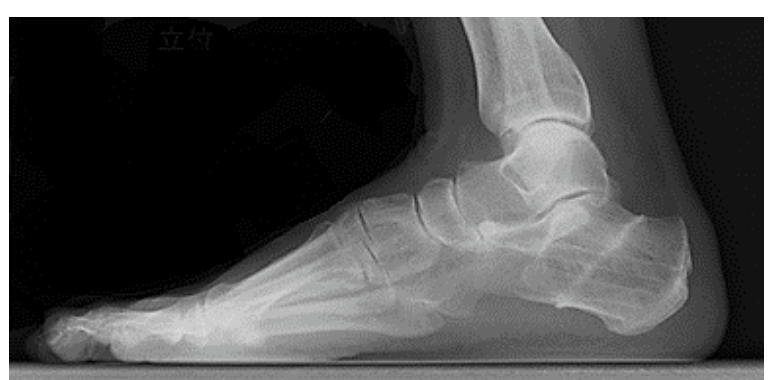

B

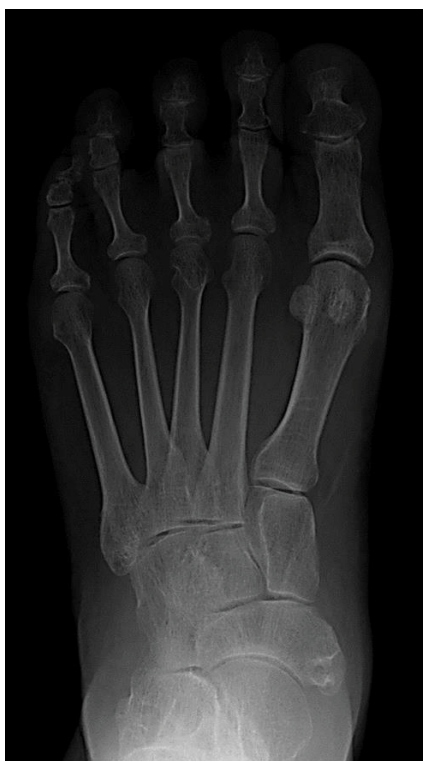

Figure 4: Radiographs at the latest follow-up A) Lateral view; B) Dorsoplantar view.

flexible flatfoot with morphological changes and loss of posterior tibial tendon function [5]. However, "active flatfoot" was not described in Myerson's classification.

The similar phenomena that were termed "active pivot shift" were reported in the case of the Anterior Cruciate Ligament $(\mathrm{ACL})$ deficient knee [8]. It was discussed that the popliteus muscle played a major role in the active pivot shift. Moreover, voluntary anterior subluxation of the proximal tibia was also reported and this was caused by a work of gastrocnemius muscle [9]. These pathological subluxations of the knee occurred by higher action of the antagonist muscles in the $\mathrm{ACL}$ deficient knee. A similar mechanism would occur in the active flatfoot.

The active flatfoot mechanism was speculated as follows. In dorsiflexion of the ankle, the tibialis anterior muscle, extensor digitalis longus and brevis muscles, the tibialis posterior muscle, and peroneus longus and brevis muscles work cooperatively. In the present patient, peroneus muscles acted so predominantly in the dysfunction of the tibialis posterior muscle that the forefoot abducted and hind foot everted, and the flatfoot deformity occurred with crepitus. After reconstruction of the posterior tibial tendon, this phenomenon disappeared. This may show support for the author's speculations, although more precise evaluation such as electromyography examination might be needed.

\section{Conclusion}

Although PTTD is a common disease, at first we could not identify this phenomenon by visual inspection, palpation, and usual imaging examination. In order to diagnose an accurate pathology, it is necessary to make dynamic examination with understanding this phenomenon.

\section{References}

1. Pomeroy GC, Pike RH, Beals TC, Manoli A (1999) Acquired flatfoot in adults due to dysfunction of the posterior tibial tendon. J Bone Joint Surg Am 81: 1173-1182.

2. DiPaola M, Raikin SM (2007) Tendon transfers and realignment osteotomies for treatment of stage II posterior tibial tendon dysfunction. Foot Ankle Clin 12: 273-285.

3. Roth S, Roth A, Jotanovic Z, Madarevic T (2013) Navicular index for differentiation of flatfoot from normal foot. Int Orthop 37: 1107-1112.

4. Johnson KA, Strom DE (1989) Tibialis posterior tendon dysfunction. Clin Orthop Relat Res 196-206.

5. Myerson MS (1997) Adult acquired flatfoot deformity: Treatment of dysfunction of the posterior tibial tendon. Instr Course Lect 46: 393-405.

6. Niki H, Aoki H, Inokuchi S, Ozeki S, Kinoshita M, et al. (2005) Development and reliability of a standard rating system for outcome measurement of foot and ankle disorders I: Development of standard rating system. J Orthop Sci 10: 457-465.

7. Niki H, Aoki H, Inokuchi S, Ozeki S, Kinoshita M, et al. (2005) Development and reliability of a standard rating sys- 
tem for outcome measurement of foot and ankle disorders II: Interclinician and intraclinician reliability and validity of the newly established standard rating scales and Japanese Orthopaedic Association rating scale. J Orthop Sci 10: 466474.

8. Peterson L, Pitman MI, Gold J (1984) The active pivot shift: the role of the popliteus muscle. Am J Sports Med 12: 313317.

9. Noda M, Kurosaka M, Yoshiya S, Onga T, Mizuno K (2000) Voluntarily evoked positive Lachman test produced by gastrocnemius muscle contraction. A report of three cases. Am J Sports Med 28: 893-896. 